\title{
Olfactory Ensheathing Glia: Drivers of Axonal Regeneration in the Central Nervous System?
}

\author{
M. Teresa Moreno-Flores, ${ }^{*}$ Javier Díaz-Nido, Francisco Wandosell, and Jesús Avila \\ Centro de Biología Molecular "Severo Ochoa" (CSIC-UAM), Facultad de Ciencias, \\ Universidad Autónoma de Madrid, 28049 Madrid, Spain
}

\begin{abstract}
Olfactory ensheathing glia (OEG) accompany olfactory growing axons in their entry to the adult mammalian central nervous system (CNS). Due to this special characteristic, considerable attention has been focused on the possibility of using OEG for CNS regeneration. OEG present a large heterogeneity in culture with respect to their cellular morphology and expressed molecules. The specific characteristics of OEG responsible for their regenerative properties have to be defined. These properties probably result from the combination of several factors: molecular composition of the membrane (expressing adhesion molecules as PSA-NCAM, L1 and/or others) combined with their ability to reduce glial scarring and to accompany new growing axons into the host CNS. Their capacity to produce some neurotrophic factors might also account for their ability to produce CNS regeneration.
\end{abstract}

\section{INTRODUCTION}

The olfactory system has remarkable distinctive properties within the adult mammalian central nervous system. Throughout the whole life of an organism, olfactory sensory neurons are renewed from progenitor cells present in the olfactory neuroepithelium (reviewed in [1,2]). New-born sensory neurons extend new axons that grow and enter the central nervous system (CNS) to make their appropriate connections in the glomerular layer of the olfactory bulbs. These olfactory axons are surrounded by a special type of glial cells called olfactory ensheathing glia (OEG), which were first described by the histologists Golgi and Blanes Viale at the end of the nineteenth century $[3,4]$. It is generally accepted that OEG are derived from the olfactory placode during development [5], although there has been some recent controversy about this [6]. In the adult olfactory system, there is no hard evidence of new OEG formation. After a lesion of the adult olfactory neuroepithelium, the mucosal neuroepithelial stem cells can repopulate it, and some OEG-like cells accompany olfactory axons in their journey from the neuroepithelium to the olfactory bulb. However, the origin of these OEG-like cells is still uncertain. There is also some doubt about the relationship between OEG present in vivo in the olfactory system and their cultured counterparts (see [6]).

From the studies of Cajal, it is known that CNS neurons have very little capacity to regenerate [7]. In contrast, neurons from the peripheral nervous system (PNS) have a notable regeneration ability that may be due, among other factors, to the peculiar nature of the Schwann cells (SC) that ensheath the PNS axons. This finding has stimulated the use of peripheral nerve and SC grafts to foster regeneration in CNS, with promising results (reviewed in [8]). Nevertheless, the incomplete CNS regeneration achieved with SC makes it necessary to search for more effective regeneration mediators. During the last few years the use of OEG for CNS regeneration has received plenty of attention, due to their special properties [6]. Since OEG usually accompany growing olfactory axons into the adult mammalian CNS, it is reasonable to expect that they may facilitate axonal regeneration. However, one fundamental question remains to be answered: do OEG permit axonal regeneration because they act as a passageway, merely encompassing and accompanying growing axons or do they play a far more active role, driving axons through hostile territory? It is not easy to answer this, but simply posing the question can help us shed light on this area. A combination of several factors, including adhesion of membrane molecules, influence on glial scarring, ability to migrate within the CNS and production of neurotrophic factors (see Figure 1), lead us to think that OEG do not act as mere passageways or accompanists but are in fact drivers of axonal regeneration in the CNS.

\section{OLFACTORY ENSHEATHING GLIA: ISOLATION AND CULTURE}

In vivo OEG express several markers that have been used for their identification after isolation and culture (see [9] and the references therein). OEG share some properties with SC and astrocytes (revised in [10]), although because of their different pattern of markers and properties are classified as a different type of glial cells.

OEG are usually isolated from the olfactory bulb of rodents. The nerve fiber layer of the olfactory bulb is dissected and the tissue, free of meninges, is removed. Then, OEG are purified using one of a number of different techniques. These include the sorting of cells positive for the sulfatide $\mathrm{O} 4$ and negative for the galactocerebroside [11], 
immunopurification procedures, selection of positive cells for the low affinity nerve growth factor receptor (p75-NGFR) or elimination of Thy-1.1 positive fibroblasts $[12,13]$, and several attachment steps based on differential adhesion of OEG [14]. Even these purified OEG cells present heterogeneous morphology and markers in culture, which mainly depends on the age of the animals used to obtain the OEGs, the time of culture, and the culture medium (unpublished observations, 2002 and [15]).

The age of the animals used to obtain the olfactory bulbs to establish the OEG culture is a major influencing factor of OEG phenotype in culture. Cultured OEG from neonate rats are nestin-positive whereas cultured OEG from adult animals are nestin-negative (see [9] and the references therein). Ran2 has also been reported to be variable in cultured OEG from neonate or adult animals $[11,12]$.

Additionally, the expression of different molecules is strongly dependent on the time and method of culture. Sorting by $\mathrm{O} 4$ sulfatide expression, a method used by some laboratories, is performed in the cells proceeding from the dissociation of olfactory bulbs to establish OEG cultures [11]. In these cultures, $\mathrm{O} 4$ decreased with culture time and was lost in serum-containing medium [15]. In our laboratory, we obtained OEG cultures without initially selecting for any molecule. Our OEG cultures maintained the expression of O4 throughout the culture time, even in the presence of serum, although the level of expression was variable from cell to cell (unpublished observations, 2002). This discrepancy did not seem to be due exclusively to the difference in the ages of the animals used, because Franceschini and Barnett reported a similar pattern of $\mathrm{O} 4$ expression in postnatal olfactory bulb, with an increase of $\mathrm{O} 4$ at the border between the olfactory nerve fiber layer and the glomerular layer in adult rats [15].

Other important OEG markers either increase (p75NGFR) or decrease (PSA-N-CAM) in a variable level or even behave in a different way (GFAP) throughout the culture time depending on the presence of serum in the medium [15].

Not all OEG markers show this variable behaviour in culture. For instance, OEG express N-CAM, S100, and vimentin independently of culture age or condition (see [9] and the references therein).

At least two OEG variants have been identified in cultures $[12,13,16]$. In serum-free medium Franceschini and Barnett have identified two extreme morphological types in the OEG cultures: an astrocyte-like flat cell that expresses GFAP (fibrous staining) and PSA-NCAM and is negative for p75-NGFR, and a second type, a Schwann-like spindle cell, expressing p75-NGFR, with diffuse staining for GFAP and negative for PSA-NCAM. All the intermediate phenotypes are possible and the observation of these phenotypes even in an established clonal cell line supports the view that they can derive from a common precursor [15]. The complementary expression of PSA-N-CAM and p75-NGFR has its equivalence in vivo, since the olfactory bulb presents areas of complementary expression for both markers during development [15]. In our OEG cultures, we have also detected both morphological types of cells and the intermediate phenotypes (unpublished observations, 2002).

The availability of cells in culture is essential for the use of OEG in grafting to favour axonal regeneration in the CNS. The OEG source and their capacity of division in primary cultures are limiting, especially when considering human CNS regeneration. Furthermore, the heterogeneity of primary OEG may not be a desirable factor for its characterisation and for its use in animal regeneration models. Thus, immortalisation and establishment of clonal cell lines, constitutes a feasible approach. Nowadays, several OEG lines, obtained by different methods, have been described. They express markers in a similar way to primary OEG (see [9] and the references therein). However, immortalising human OEG remains an important step to establish primate models of CNS regeneration, which may lead to novel therapeutic interventions in humans.

\section{OEG and growth factors}

One of the most important requisites for the use of these cells is a complete understanding of their growth requirements. Certain mitogens for OEG were initially characterised as factors present in astrocyte conditioned medium (ACM), that do not bind to heparin and can be inhibited with antibodies against neuregulin-1 (NRG-1) [17]. Moreover, semipurified bovine pituitary extract, which is a crude source of several glial mitogens including GGF a NRG-1, is active for OEG [17]. In fact, GGF2 has been shown to promote OEG proliferation [18]. Thus, NRG-1 proteins are important mitogen and survival factors for OEG; and probably a secreted type III NRG-1 is present in ACM [17]. The neuregulin family of growth factors comprises a group of molecules that share an EGF-like domain and are encoded by 4 different genes. NRG-1 gene presents 13 exons and encodes for multiple isoforms that can be divided in three types: I (NDF/heregulin or ARIA), II (GGF), and III (SMDF), depending on their N-terminal sequences (for review see [19]). NRG-1s may be transmembrane or secreted proteins. All types are expressed by SC, astrocytes, and OEG. However, in contrast to SC and astrocytes, OEG do not secrete significant amounts of NRG-1 (see [20] and the references therein).

ErbB-3 and ErbB-4 are preferential receptors for NRG1-4. Pollock and coworkers have demonstrated that cultured OEG present ErbB-2 and 4 receptors, lacking ErbB-3 [17]. We have found, by Western blot analysis, that OEG express not only ErbB-2 and 4 but also ErbB-3 (unpublished observations, 2002). The level of expression of ErbB receptors by OEG was higher for ErbB-2 and 3 than for ErbB-4, and depends on the culture medium, being increased in the presence of forskolin and pituitary extract (unpublished observations, 2002). ErbB-3 and ErbB-4 form homo- or heterodimers by recruiting ErbB-2 coreceptor to transduce NRG signals (see [19]). The functions of certain combinations of ErbB receptors for signalling in OEG remain to be determined.

In vivo, NRG transcripts have been shown in the olfactory bulb of mouse embryos [21]. In the adult olfactory system, ErbB- 2 and NRG- $\alpha 1$ are expressed by the globose basal 
cells of the neuroepithelium, the immature olfactory sensory neurons and OEG of the olfactory nerve [22]; ErbB-3 by non-neuronal cells of the olfactory nerve [23], and ErbB-4 by the periglomerular and the mitral/tufted cells [24]. The levels of ErbB2 and ErbB-4/neuregulin-1 are inversely regulated in the olfactory bulb after peripheral denervation. Expression of ErbB-4 decreases after lesion, possibly as a consequence of peripheral olfactory innervation. The correlation with the regulation of neuregulin-1 levels suggests a possible functional link [24]. The intercellular relationship in the olfactory system with respect to NRG and ErbB cross talking requires further research.

Pituitary extract and stimulation of cAMP pathway (ie, cholera toxin or forskolin) were previously used in the protocols to grow SC. However, the mechanisms by which forskolin increases the response of SC to growth factors are poorly understood. Only for PDGF has it been shown that forskolin promotes the expression of their receptors [25] but it is not clear whether it affects the expression of other mitogen receptors or modulates intracellular signalling pathways. Our group has also used this system to promote proliferation of cultured adult OEG. A recent work has described that heregulin (neuregulin-1 type I), FGF-2, PDGF$\mathrm{BB}$, and IGF-1 are able to stimulate OEG proliferation in serum-containing medium; and that this effect is increased by forskolin [26]. The combination of heregulin with all the other factors and the combination of FGF-2 with both PDGF-BB and IGF-1 promoted OEG proliferation in an additive way. However, in the absence of serum, exclusively heregulin and FGF-2 were mitogenic for adult cultured OEG. Forskolin stimulated this effect and both factors had an additive effect on OEG mitosis [26]. Moreover, FGF-2 was found to be mitogenic for neonatal OEG in culture [27], and both mitogenic and promotor of differentiation for olfactory sensory neurons [28]. Thus, FGF-2 might exert a function in the olfactory system, but its cellular source there remains to be determined.

\section{PROMOTION OF NEURITE OUTGROWTH BY OEG}

Cultured OEG present a large heterogeneity with respect to their morphological and molecular properties. It is necessary to fully understand the neuritogenic properties of OEG to improve their application in models in vivo of CNS injuries. To this aim, characterization of cell adhesion molecules, neurotrophic factors, and extracellular matrix proteins expressed by OEG is essential. Likewise, it would also be useful to establish in vitro models to study OEGinduced neurite growth.

\section{Cell adhesion molecules, neurotrophic factors, and extracellular matrix proteins}

The specific features of OEG responsible for their axonal regeneration-promoting properties have yet to be determined. These properties can probably be attributed to a combination of several factors. The molecular composition of the membrane, in particular the presence of cell adhesion molecules such as PSA-NCAM, L1 and/or others, their capacity to secrete some neurotrophic factors and the ability to produce neuritogenic extracellular matrix proteins might account for the regenerative capacity of OEG (see Figure 1).

In vivo, the olfactory bulb shows expression of N-CAM throughout the nerve fiber layer, and a more restricted expression of the embryonic form of N-CAM, PSA-NCAM, which presents a complementary expression pattern to that of p75-NGFR during development [15]. OEG in vivo also express L1 (see [9] and the references therein). However, in culture, only fragmentary information is available. Cultured OEG from neonatal and P7 rats express N-CAM, and some astrocyte-like cells express PSA-NCAM depending on the culture conditions $[13,15]$ (see the previous section). Cultured OEG from adult rat brains express L1 [12, 29]. However, it is still necessary to determine the complete pattern of expression of adhesion molecules by OEG and their correlation with OEG regenerative properties.

Additionally, neurotrophins might play an important role in the olfactory system. Neonatal OEG express and secrete NGF and BDNF, although NGF is more abundant [30]. OEG also present the neurotrophin receptor trkB but lack trkA and trkC. Thus, OEG are a source of neurotrophins in the olfactory system. Some experimental evidence supports an important role for neurotrophins in the survival, proliferation, and differentiation of olfactory neurons (see [30]). The fact that OEG also express trkB implies that BDNF might have an autocrine effect on them. In addition, OEG express GDNF, neurturin (NTN) and their respective receptors GFR $\alpha-1$ and GFR $\alpha-2$, but not the transducing receptor RET [30]. GFR $\alpha$ is present in the olfactory epithelium and in the olfactory bulb. It has been hypothesised that OEG, lacking RET and thus unable to transduce the signal, may bind and present GDNF and NTN to growing neurons, but their function in the olfactory system is still unknown [30].

The ability of OEG to produce certain neurotrophic factors might account for the capacity of OEG grafts in lesioned spinal cord to facilitate the regeneration of some spinal tracts but not others, in function of their trophic requirements (see [8]).

In the olfactory system, OEG express laminin, an extracellular matrix protein that is highly neuritogenic (see [9] and the references therein). Cultured OEG from adult rats also express laminin [12].

\section{Tissue culture models of OEG-induced axonal regeneration}

The ability of OEG to promote neurite outgrowth can be used to establish neuritogenesis and/or regeneration models in culture. Neurite outgrowth in culture may simply indicate neuritogenesis when using embryonic or neonatal neurons. However, neurite extension from adult CNS neurons can be considered as a culture model of CNS regeneration. Thus, Wigley and Berry established a coculture model using adult retinal ganglion cells (RGC) on a monolayer of glial cells. Adult RGC cultured on a confluent monolayer of neonatal cortical astrocytes could regrow neurites over long distances 


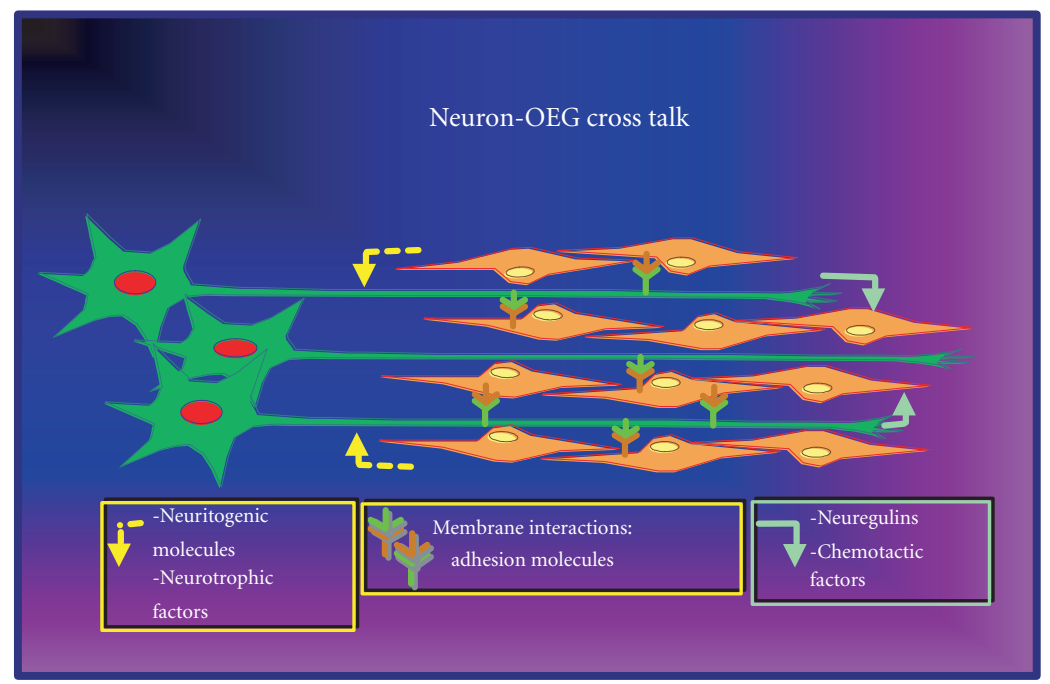

FIGURE 1. Neuron-OEG cross talk. OEG and extending axons of olfactory sensory neurons interact through membrane adhesion molecules (represented by the Y symbols). OEG also bear receptors to neuregulins and possibly to other chemotactic factors produced by the developing neurons (solid arrows) and/or other cells. Furthermore, OEG produce and secrete neurotrophic factors and neuritogenic extracellular matrix proteins which interact with developing neurons (dashed arrows). The characteristics of OEG together with their influence on glial scarring and their ability to migrate within the CNS might be responsible for their axonal-regenerative properties in CNS injuries. OEG permit CNS axonal regeneration by acting as a vehicle, ensheathing, driving, and nurturing growing axons through a hostile territory.

[31]. In a recent study, the group of Wigley, using the same model, compared monolayers of adult rat OEG, to neonatal astrocytes and SC. They demonstrated that adult OEG were the best substrate for neurite regeneration of adult RGC in culture, and that this effect mainly depended on intercellular contact and calcium [32]. In fact SC-conditioned medium (CM), but not OEG-CM, was a source of neurotrophic factors [32]. In contrast, embryonic OEG that are capable of promoting neurite extension of embryonic olfactory receptor cells, are a significant source of diffusible neurotrophic factors, present in the OEG-CM [33]. The difference between both systems may be either due to the age of the animals from which OEG cultures were derived or to the different types of neurons used in the cocultures. Cellular surface molecules (factors for adhesion, axonal guiding, etc) are important for adult RGC as demonstrated by Sonigra and coworkers, but their exact identity and their role in other neurons should be tested [32]. On the other hand, they could not find any evidence for the secretion of neurotrophic factors for RGC by adult OEG. However, the different trophic requirements of diverse types of neurons could account for the differences between RGC and olfactory sensory neurons. It is also possible that embryonic or neonatal OEG express and/or secrete neurotrophic factors differently to adult OEG. Neonatal OEG have been shown to express and secrete NGF, BDNF and to express GDNF and NTN (see above, and [30]), yet expression of neurotrophic factors by adult OEG remains to be demonstrated.

It would be naïve to think of a simple correspondence between adhesion molecules or neurotrophic factors and axonal regeneration. Probably, a balance of all these factors is responsible for optimal effects.

\section{PROMOTION OF AXONAL REGENERATION IN VIVO BY OEG}

Axonal regeneration in the CNS is a complex issue that must be considered from a broad point of view and requires an integral approach (for review see [34]). PNS presents an important regeneration capacity, but this is not the case for CNS, which has very little ability to regenerate. The pioneering studies of the group of Aguayo and other groups using peripheral nerve grafting support the idea that CNS neurons can regenerate axons provided that they are in a stimulatory environment [35]. However, more recent studies also give more weight to the inherent capacity of neurons to regenerate or not. This is a function of the expression of genes necessary for axonal regrowth [36]. It is clear that, after CNS injury, even if the neurons that remain alive are capable of axonal regrowth, these new axons should navigate in a permissive molecular background and be properly guided if they are to resynapse with suitable targets.

Experimental evidence points to a fundamental role for the CNS microenvironment in preventing regeneration after a lesion. Several factors may account for this; for example, after a CNS lesion, a glial reaction occurs, which gives way to the formation of a "glial scar." Several molecules present in this scar tissue are inhibitors for axonal growth, including myelin and some of their components (NOGO and myelin associated glycoprotein, MAG), some proteoglycans, and other negative factors such as the axonal guiding proteins semaphorin 3A and EphB3 [37, 38, 39]. Additionally, axonal growth also fails because of deficient neurotrophic factors and/or the absence of positive axonal guiding signals. 
Accordingly, manipulation of the negative microenvironment of the injured CNS may hopefully turn it into a permissive one. Initially, regeneration in the CNS has been achieved by the use of PNS SC grafts (revised in [8]). CNS regrowing axons can penetrate this permissive environment but a problem is posed by the low efficiency of reentry into CNS tissue distal to the lesion. Previous experiments by the group of Manuel Nieto-Sampedro indicated that OEG could mediate reentry of peripheral sensory axons in spinal cord CNS environment after rizhotomy [29]. Reentry of regenerating axons into the CNS has also been observed after a complete transection of the spinal cord, when a bridge tube with SC and OEG was placed. The presence of OEG decreased the formation of the glial scar and constituted an axonal passageway at the graft-host interface [40]. This method provided a way of increasing the efficiency of reentry of regenerating axons in the CNS host tissue. The group of Raisman also showed recovery of the corticospinal tract function after OEG grafting in partially transected spinal cord [41]. Using the model of rat spinal cord complete transection, an extraordinary degree of CNS regeneration affecting several tracts of the spinal cord, has been demonstrated, with accompanying functional recovery measured by behavioral tests [42]. OEG seem to be able to migrate in the host CNS tissue and to go along with the growing axons thus providing a favorable microenvironment for CNS regeneration $[42,43]$.

\section{PROSPECTS FOR THE FUTURE: USE OF OEG GRAFTS FOR HUMAN CENTRAL NERVOUS SYSTEM REGENERATION}

Initially, we formulated a fundamental question: do OEG act as a passageway, ensheathing growing axons, accompanying them, and being driven by them through a CNS lesion; or are they active drivers which promote the growth of these axons through a hostile territory? Or ultimately, do OEG have a passive or an active role in CNS regeneration? Before we can answer these questions and consider using OEG for human CNS regeneration in the future, we will have to deepen our understanding of their normal physiology, especially of control of their proliferation and migration and the molecules responsible for their neuritogenic capacity. Nevertheless, the special characteristics of OEG suggest that they permit axonal regeneration because they act as a vehicle, ensheathing and also driving and nurturing growing axons through a hostile territory.

Spinal cord injury is a particularly serious lesion that makes normal life completely impossible. Moreover, a large number of severe CNS injuries must also be taken into account. It is also worth noting that several studies have shown that OEG can myelinate axons (see [44] and references therein) opening up the possibility of using them in demyelinating diseases.

Previous experiments with engineered SC and fibroblasts expressing different neurotrophic factors have been useful in helping to repair spinal cord lesions, particularly certain nerve tracts $[8,45,46]$. The separation of relevant information for CNS repair from "noise" will permit us to determine the fundamental factors required for OEG-induced axonal regeneration and the possibility of studying further modifications to optimise the repair of different CNS injuries.

Whereas experiments performed in rodents suggest that OEG grafts favor functional recovery after spinal cord injuries $[41,42]$, these studies must be extended to primates, in which the supraspinal control of motor functions is fundamental to assess functional recovery. Of the utmost importance in this respect is the availability of an accessible and nonlimited source of human OEG for grafting into patients. The generation of immortalised nontumorigenic clonal cell lines, which retain the axonal regeneration-promoting properties of primary OEG cultures, is an obvious possibility. Alternatively, the isolation of OEG progenitors (or stem cells) and the control of their differentiation into axonal-growthpromoting and ensheathing cells may also be worth considering. Hopefully, we are closer than ever to obtaining effective axonal regeneration after human CNS injuries to achieve functional recovery. However, a huge amount of work, presumably integrating different experimental strategies, including the use of OEG, is required before we can approach this long-sought aim.

\section{ACKNOWLEDGMENTS}

Our work was supported by grants of DGCYYT and Neuropharma. M. Teresa Moreno-Flores was supported by spanish CSIC and Neuropharma contracts.

\section{REFERENCES}

[1] Farbman AI. Olfactory neurogenesis: genetic or environmental controls? Trends Neurosci. 1990;13:362-365.

[2] Doucette R. Glial cells in the nerve fiber layer of the main olfactory bulb of embryonic and adult mammals. Microsc Res Tech. 1993;24:113-130.

[3] Golgi C. Sulla fina anatomia del bulbi olfattorii. Ti Rivista Sperimentale di Freniatria. 1875;1:403-425.

[4] Blanes T. Sobre algunos puntos dudosos de la estructura del bulbo olfatorio. Rev Trim Micrograf. 1898;3:99-127.

[5] De Carlos JA, Lopez-Mascaraque L, Valverde F. Early olfactory fiber projections and cell migration into the rat telencephalon. Int J Dev Neurosci. 1996;14:853-866.

[6] Raisman G. Olfactory ensheathing cells-another miracle cure for spinal cord injury? Nat Rev Neurosci. 2001;2:369-375.

[7] Ramón y Cajal S. Degeneration and Regeneration of the Nervous System. Translated by May, RM. London: Oxford University Press; 1928

[8] Jones LL, Oudega M, Bunge MB, Tuszynski MH. Neurotrophic factors, cellular bridges, and gene therapy for spinal cord injury. J Physiol. 2001;533:83-89.

[9] Ramón-Cueto A, Ávila J. Olfactory ensheathing glia: properties and function. Brain Res Bull. 1998;46:175187.

[10] Doucette R. Glial influences on axonal growth in the primary olfactory system. Glia. 1990;3:433-449. 
[11] Barnett SC, Hutchins AM, Noble M. Purification of olfactory nerve ensheathing cells from the olfactory bulb. Dev Biol. 1993;155:337-350.

[12] Ramón-Cueto A, Nieto-Sampedro M. Glial cells from adult rat olfactory bulb: immunocytochemical properties of pure cultures of ensheathing cells. Neuroscience. 1992;47:213-220.

[13] Chuah MI, Au C. Olfactory cell cultures on ensheathing cell monolayers. Chem Senses. 1994;19:25-34.

[14] Nash HH, Borke RC, Anders JJ. New method of purification for establishing primary cultures of ensheathing cells from the adult olfactory bulb. Glia. 2001;34:81-87.

[15] Franceschini IA, Barnett SC. Low-affinity NGFreceptor and E-N-CAM expression define two types of olfactory nerve ensheathing cells that share a common lineage. Dev Biol. 1996;173:327-343.

[16] Pixley SK. The olfactory nerve contains two populations of glia, identified both in vivo and in vitro. Glia. 1992;5:269-284.

[17] Pollock GS, Franceschini IA, Graham G, Marchionni MA, Barnett SC. Neuregulin is a mitogen and survival factor for olfactory bulb ensheathing cells and an isoform is produced by astrocytes. Eur J Neurosci. 1999;11:769-780.

[18] Chuah MI, Cossins J, Woodhall E, Tennent R, Nash G, West AK. Glial growth factor 2 induces proliferation and structural changes in ensheathing cells. Brain Res. 2000;857:265-274.

[19] Buonanno A, Fischbach GD. Neuregulin and ErbB receptor signaling pathways in the nervous system. Curr Opin Neurobiol. 2001;11:287-296.

[20] Thompson RJ, Roberts B, Alexander CL, Williams SK, Barnett SC. Comparison of neuregulin-1 expression in olfactory ensheathing cells, Schwann cells and astrocytes. J Neurosci Res. 2000;61:172-185.

[21] Meyer D, Birchmeier C. Distinct isoforms of neuregulin are expressed in mesenchymal and neuronal cells during mouse development. Proc Natl Acad Sci USA. 1994;91:1064-1068.

[22] Salehi-Ashtiani K, Farbman AI. Expression of neu and Neu differentiation factor in the olfactory mucosa of rat. Int J Dev Neurosci. 1996;14:801-811.

[23] Perroteau I, Oberto M, Soncin I, et al. Transregulation of erbB expression in the mouse olfactory bulb. Cell $\mathrm{Mol}$ Biol (Noisy-le-grand). 1999;45:293-301.

[24] Oberto M, Soncin I, Bovolin P, et al. ErbB-4 and neuregulin expression in the adult mouse olfactory bulb after peripheral denervation. Eur J Neurosci. 2001;14:513-521.

[25] Weinmaster G, Lemke G. Cell-specific cyclic AMPmediated induction of the PDGF receptor. EMBO J. 1990;9:915-920.

[26] Yan H, Bunge MB, Wood PM, Plant GW. Mitogenic response of adult rat olfactory ensheathing glia to four growth factors. Glia. 2001;33:334-342.

[27] Chuah MI, Teague R. Basic fibroblast growth factor in the primary olfactory pathway: mitogenic effect on ensheathing cells. Neuroscience. 1999;88:1043-1050.
[28] MacDonald KP, Murrell WG, Bartlett PF, Bushell GR, Mackay-Sim A. FGF2 promotes neuronal differentiation in explant cultures of adult and embryonic mouse olfactory epithelium. J Neurosci Res. 1996;44: 27-39.

[29] Ramón-Cueto A, Nieto-Sampedro M. Regeneration into the spinal cord of transected dorsal root axons is promoted by ensheathing glia transplants. Exp Neuro. 1994;127:232-244.

[30] Woodhall E, West AK, Chuah MI. Cultured olfactory ensheathing cells express nerve growth factor, brainderived neurotrophic factor, glia cell line-derived neurotrophic factor and their receptors. Brain Res Mol Brain Res. 2001;88:203-213.

[31] Wigley CB, Berry M. Regeneration of adult rat retinal ganglion cell processes in monolayer culture: comparisons between cultures of adult and neonatal neurons. Brain Res. 1988;470:85-98.

[32] Sonigra RJ, Brighton PC, Jacoby J, Hall S, Wigley CB. Adult rat olfactory nerve ensheathing cells are effective promoters of adult central nervous system neurite outgrowth in coculture. Glia. 1999;25:256-269.

[33] Kafitz KW, Greer CA. Olfactory ensheathing cells promote neurite extension from embryonic olfactory receptor cells in vitro. Glia. 1999;25:99-110.

[34] Horner PJ, Gage FH. Regenerating the damaged central nervous system. Nature. 2000;407:963-970.

[35] Bray GM, Villegas-Perez MP, Vidal-Sanz M, Carter DA, Aguayo AJ. Neuronal and nonneuronal influences on retinal ganglion cell survival, axonal regrowth, and connectivity after axotomy. Ann N Y Acad Sci. 1991;633:214-228.

[36] Bomze HM, Bulsara KR, Iskandar BJ, Caroni P, Skene JH. Spinal axon regeneration evoked by replacing two growth cone proteins in adult neurons. Nat Neurosci. 2001;4:38-43.

[37] Fawcett JW, Asher RA. The glial scar and central nervous system repair. Brain Res Bull. 1999;49:377-391.

[38] Pasterkamp RJ, Verhaagen J. Emerging roles for semaphorins in neural regeneration. Brain Res Brain Res Rev. 2001;35:36-54.

[39] Miranda JD, White LA, Marcillo AE, Willson CA, Jagid J, Whittemore SR. Induction of Eph B3 after spinal cord injury. Exp Neurol. 1999;156:218-222.

[40] Ramón-Cueto A, Plant GW, Ávila J, Bunge MB. Longdistance axonal regeneration in the transected adult rat spinal cord is promoted by olfactory ensheathing glia transplants. J Neurosci. 1998;18:3803-3815.

[41] Li Y, Field PM, Raisman G. Repair of adult rat corticospinal tract by transplants of olfactory ensheathing cells. Science. 1997;277:2000-2002.

[42] Ramón-Cueto A, Cordero MI, Santos-Benito FF, Ávila J. Functional recovery of paraplegic rats and motor axon regeneration in their spinal cords by olfactory ensheathing glia. Neuron. 2000;25:425-435.

[43] Li Y, Field PM, Raisman G. Regeneration of adult rat corticospinal axons induced by transplanted olfactory ensheathing cells. J Neurosci. 1998;18:10514-10524. 
[44] Franklin RJ, Barnett SC. Olfactory ensheathing cells and CNS regeneration: the sweet smell of success? Neuron. 2000;28:15-18.

[45] Grill R, Murai K, Blesch A, Gage FH, Tuszynski MH. Cellular delivery of neurotrophin-3 promotes corticospinal axonal growth and partial functional recovery after spinal cord injury. J Neurosci. 1997;17:5560-5572.

[46] Liu Y, Kim D, Himes BT, et al. Transplants of fibroblasts genetically modified to express BDNF promote regeneration of adult rat rubrospinal axons and recovery of forelimb function. J Neurosci. 1999;19:4370-4387.

* Corresponding author.

E-mail: mtmoreno@cbm.uam.es

Fax: +34 91 3974799; Tel: +34913978475 

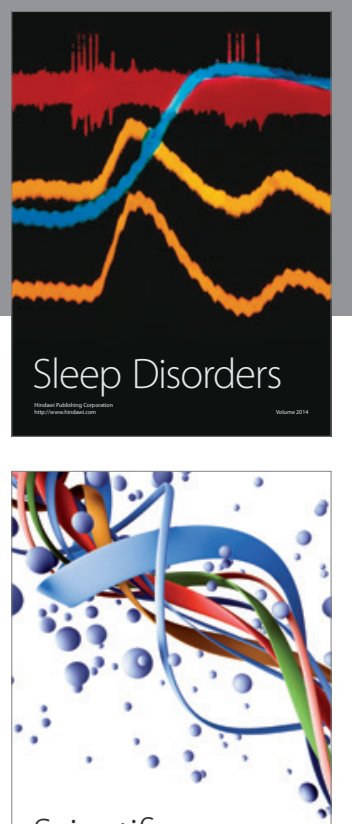

Scientifica
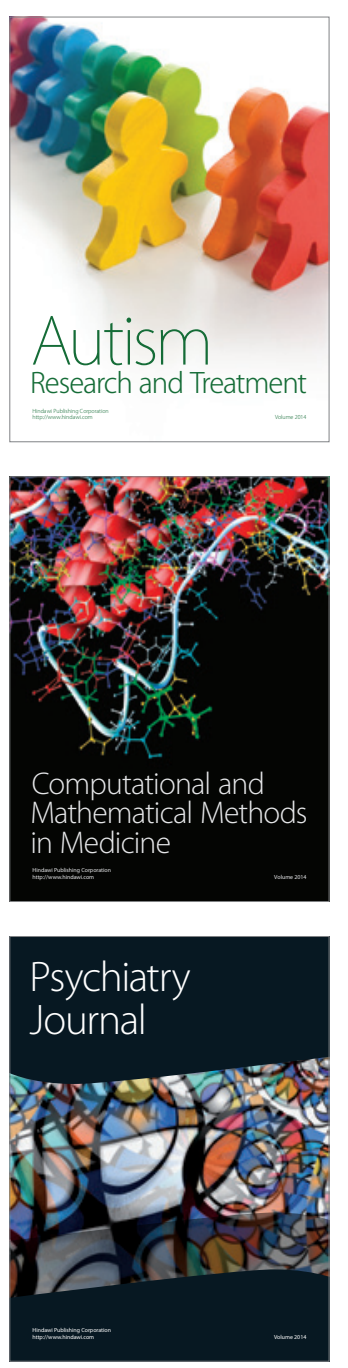
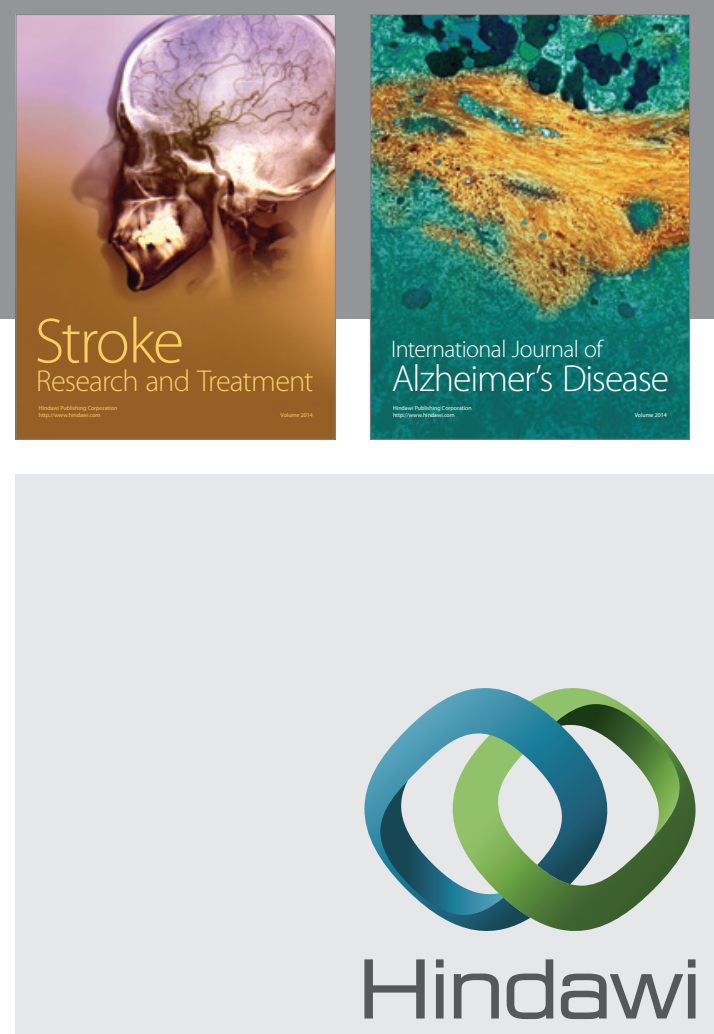

Submit your manuscripts at

http://www.hindawi.com
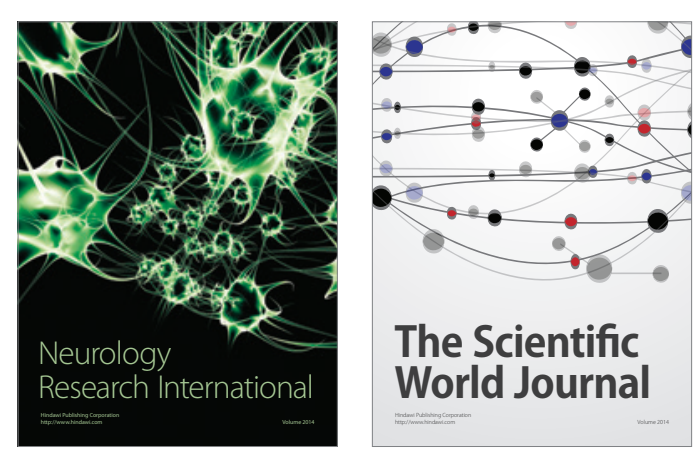

The Scientific World Journal

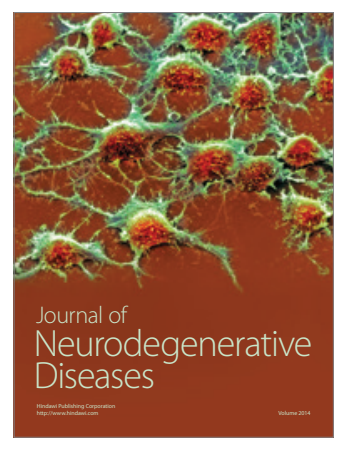

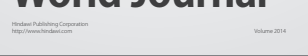

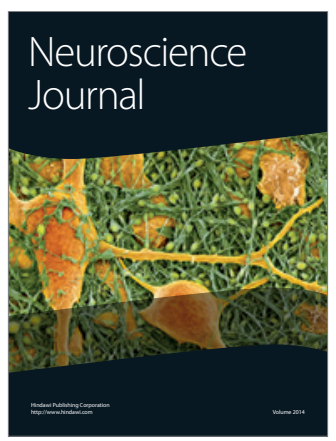

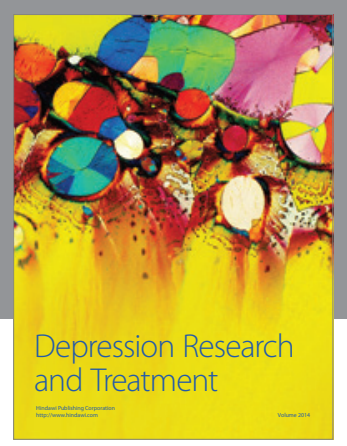
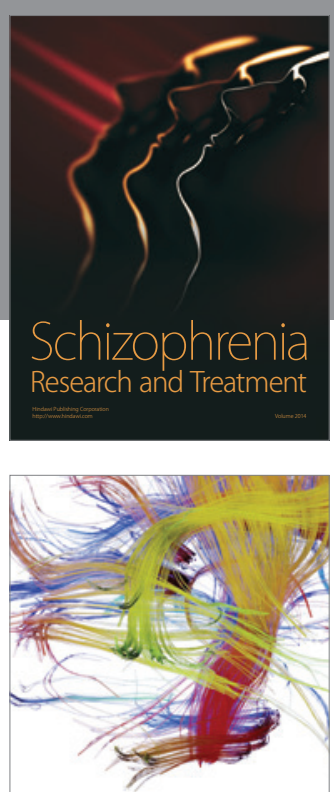

Brain Science

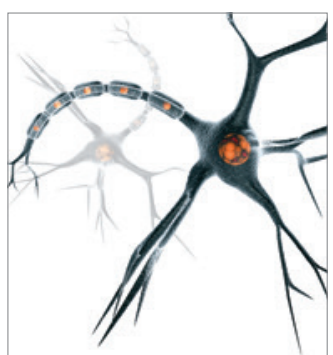

Neural Plasticity
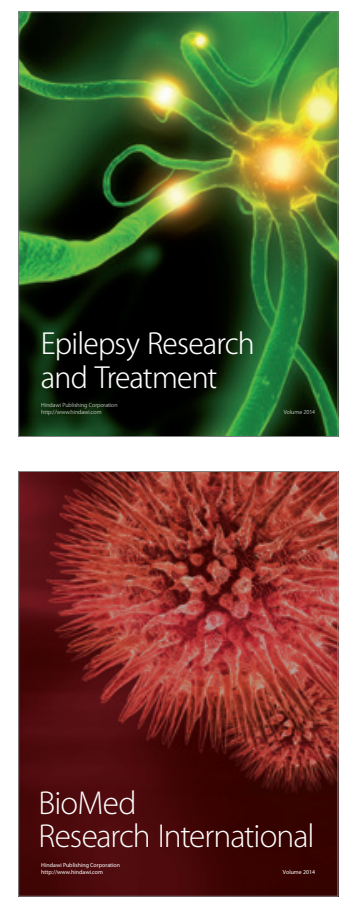

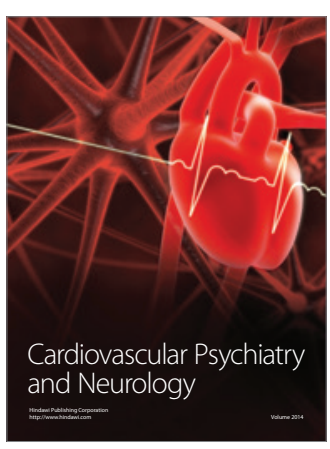

Parkinson's

Disease
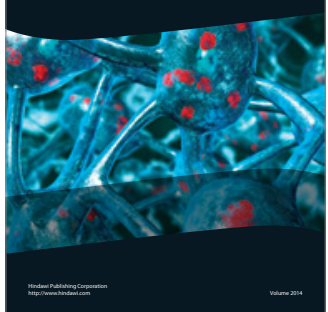